\title{
$X$-ray Diffraction Studies and Molecular Dynamics Calculations of the Structure of Alkali Disilicate Glasses
}

\author{
Nouliaki AOKI, Hiroshi HASEGAWA and Itaru YASUI \\ (Institute of Industrial Science, University of Tokyo \\ 22-1, Roppongi 7 chome, Minato-ku, Tokyo 106 )
}

\begin{abstract}
The structures of $\mathrm{K}_{2} \mathrm{O} \cdot 2 \mathrm{SiO}_{2}$ and $\mathrm{Li}_{2} \mathrm{O} \cdot 2 \mathrm{SiO}_{2}$ glasses were investigated by the X-ray diffraction measurement. Their structures were determined by the pair function analysis of the radial distribution function and by the molecular dynamics calculation (for only $\mathrm{K}_{2} \mathrm{O} \cdot 2 \mathrm{SiO}_{2}$ glass). The structures of alkali disilicate glasses were shown to consist of layers of six-membered ring of $\mathrm{SiO}_{4}$ tetrahedra with potassium or lithium ions occupying positions between them. Potassium ions and lithium ions were accomodated in the space formed by four non-bridging oxygen atoms of upper and lower layers. The layer structure of disilicate glass was recognized as a composite of chains of their corresponding metasilicate glasses. In analysis of the structure of $\mathrm{K}_{2} \mathrm{O} \cdot 2 \mathrm{SiO}_{2}$ glass, the molecular dynamics calculations were effective to obtain the most stable configuration. The result agreed with the previous study on $\mathrm{Na}_{2} \mathrm{O} \cdot 2 \mathrm{SiO}_{2}$ and $\mathrm{Li}_{2} \mathrm{O} \cdot \mathrm{K}_{2} \mathrm{O} \cdot 4 \mathrm{SiO}_{2}$ glasses, which were determined to have the layer structures.
\end{abstract}

[Received July 9, 1985] Key-words : Disilicate glass, Molecular dynamics calculation, Layer structure, Alkali ion, SiO,tetrahedron, Born-Mayer
potential, Minimum image convention method

\section{$\mathbf{X}$ 線回折と分子動力学によるディシリケートガラスの構造解析}

\author{
青木能理顥・長谷川 洋・安井 至 \\ (東京大学 生産技術研究所)
}

1. Introduction

It took long time and much efforts to obtain a suitable model by the method we have developed and used ${ }^{11}$. In order to cut down the time to reach the best models, we employed a new method, i. e. molecular dynamics method. Molecular dynamics calculations were effective to obtain the probable configuration with a little effort. Either molecular dynamics method or Monte Carlo technique was useful to describe the structures of liquid alkali chlorides and also vitreous silica by Woodcock and Singer ${ }^{2)}$. Also Soules ${ }^{3,4}$ ) showed instructive reports on sodium silicate glasses, a potassium-sodium silicate and soda-lime glasses. With appropriately chosen Born-Mayer parameters, the structures of alkali disilicate glasses could be obtained by molecular dynamics calculations. We chose the minimum image convension method in the calculations of the structure of potassium disilicate glass. Molecular dynamics calculations were applied to the relaxational procedure from the structure of the constructed model to that of the obtained $\mathrm{K}_{2} \mathrm{O} \cdot 2 \mathrm{SiO}_{2}$ glass. In the analysis of the structure of lithium disilicate glass, some models of network structure were made up on the basis of the method reported previously ${ }^{1,5)}$.

\section{Experimental procedure}

\section{1 Sample preparation}

In production of disilicate glasses, mixtures of reagent grade alkali carbonate and optical glass grade $\mathrm{SiO}_{2}$ in specified ratio were melted in a $\mathrm{Pt}-\mathrm{Rh}$ crucible.

In production of potassium disilicate glass, the mixture was melted at $1100^{\circ} \mathrm{C}$, then poured into a brass mold and left to cool in dry box. The measured density of potassium disilicate glasses was $2.456 \mathrm{~g} / \mathrm{cm}^{3}$. Because the potassium disilicate glass is hygroscopic, the sample was mounted in epoxy resin and one side of the free surface was polished in mineral oil. Then it was kept in a vacuum chamber during X-ray diffraction measurement. The sample was $3.0 \mathrm{~mm}$ thick, whose thickness was enough to diffract more than $99 \%$ of the incident X-ray. 
In production of lithium disilicate glass, the mixture was melted at $1350^{\circ} \mathrm{C}$ and rapidly quenched to prevent from crystallization. The obtained glass was annealed at the glass transformation temperature, $470^{\circ} \mathrm{C}$. The density was measured using a piece of glass by Archimedean method. The measured density of lithium disilicate glass was $2.345 \mathrm{~g} / \mathrm{cm}^{3}$. Lithium disilicate glass is too viscous to obtain planer glass specimen. Then the small piece of glass obtained was crushed to powder. The thickness of the sample calculated for sufficient X-ray absorption was $6.0 \mathrm{~mm}$, so powder was filled it into brass holder with $10 \mathrm{~mm}$ deep chamber.

\subsection{X-ray diffraction measurement}

The X-ray diffraction profile of each disilicate glass was obtained by using rotaflex (Rigaku Denki Co.) with rotating Mo anode with a maximum output of $50 \mathrm{kV}-100 \mathrm{~mA}$. Mo $K \alpha$ radi- ation was monochromatized with a couple of Ross's balanced filters ( $\mathrm{Zr}$ and $\mathrm{Y}$ ) and a graphite monochrometer, together with pulse height analizer and a scintillation counter. A step scanning method in fixed counting time of $800 \mathrm{~s}$ was adopted to minimize statistical errors.

The angular range of measurements was from $2 \theta=4^{\circ}$ to $150^{\circ}$ (i.e., $k=0.06$ to 17.0 ) was repeated once at lower diffraction angle or three times at higher diffraction angle in order to maintain high precision, and their average was used as the diffraction data.

After correction for polarization and air scattering, the data were normalized to electron units by taking account of a correction for multiple scattering. The experimental amplitude functions, $k_{i}(k)$, were shown in Fig. 1 . Then $k_{i}$ $(k)$ functions were Fourier-transformed to the radial distribution functions (RDF), which were shown in Fig. 2.

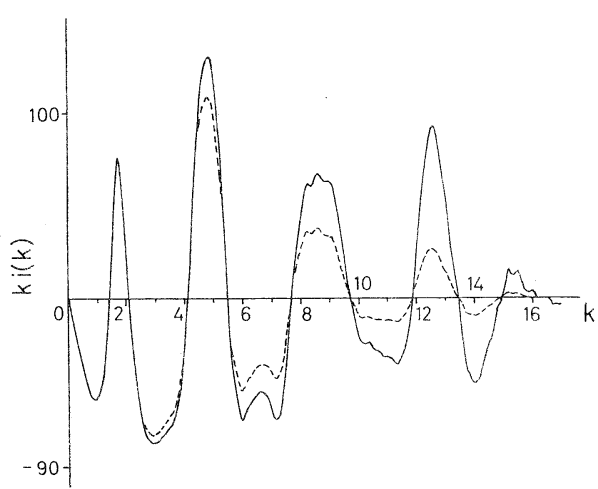

(A)

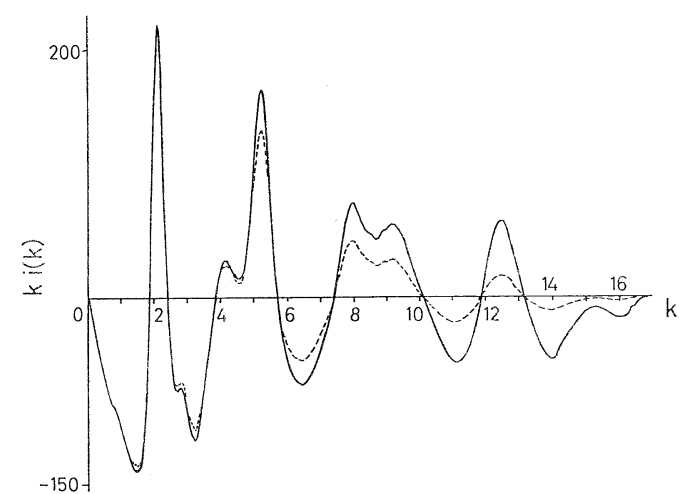

(B)

Fig. 1. Experimental amplitude functions of $\mathrm{Li}_{2} \mathrm{O} \cdot 2 \mathrm{SiO}_{2}$ glass $(\mathrm{A})$ and $\mathrm{K}_{2} \mathrm{O} \cdot 2 \mathrm{SiO}_{2}$ glass (B).

: $k_{i}(k)$

$\cdots \cdots: k_{i}(k) \exp \left(-a k^{2}\right)$, with $a=0.0079$

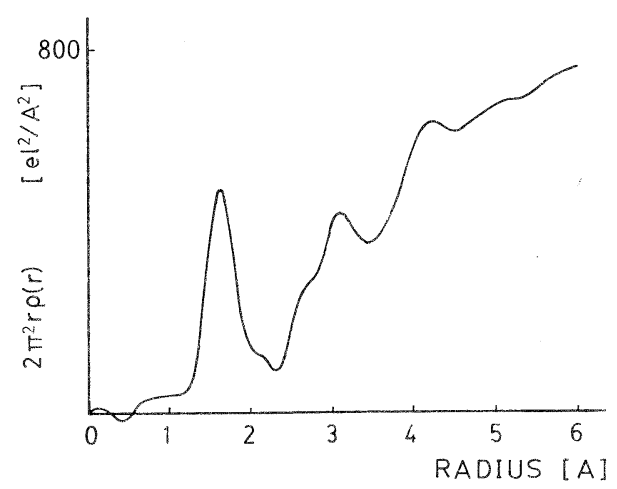

(A)

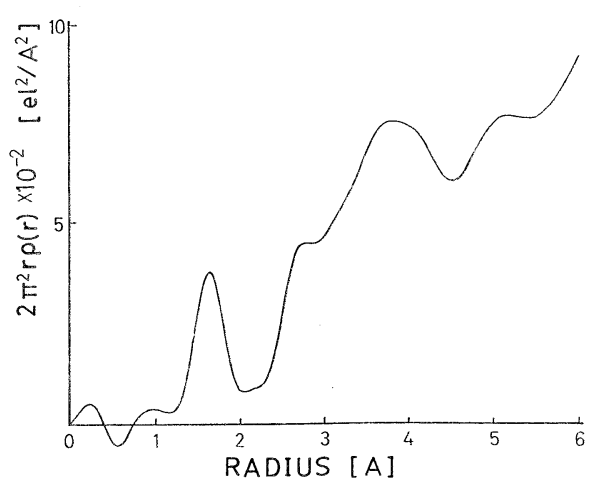

(B)

Fig. 2. Observed radial distribution function (RDF) curves of $\mathrm{Li}_{2} \mathrm{O} \cdot 2 \mathrm{SiO}_{2}$ glass (A) and $\mathrm{K}_{2} \mathrm{O} \cdot 2 \mathrm{SiO}_{2}$ glass $(\mathrm{B})$. 


\section{Molecular dynamics (MD) calculations of $\mathrm{K}_{2} \mathrm{O} \cdot 2 \mathrm{SiO}_{2}$ glass}

Molecular dynamics (MD) calculations were carried out only for the analysis of the structure of potassium disilicate glass. In the analysis of the structure of lithlum disilicate glass, its structure was obtained only by the pair function method, because we encountered difficulty in obtaining an adequate structure by the minimum image convention method.

\section{1 Method and the pairwise potentials}

$\mathrm{MD}$ calculations were performed by assigning effective interatomic potentials to the respective kinds of atomic pairs. The effective pairwise potentials are of the following form;

$$
\begin{aligned}
U_{i j}(i)= & (1 / 4 \pi \varepsilon) z_{i} z_{j} / r_{i j}{ }^{-1} \\
& +b^{*} \exp (-r / p)
\end{aligned}
$$

where $z$ is the electron charge, $\varepsilon$ is the dielectric constant in vacuum, and $b$ and $p$ are empirical contstants respectively. HugginsMayer potential was frequently used in the MD calculations. The procedure of the calculation is to intergrate the Newtonian equation of motion, and the force acting on an ion was obtained as a summation over all the pair forces by using Verlet's algorithm ${ }^{6}$. The covalency of $\mathrm{Si}-\mathrm{O}$ bonding was took into consideration, while the $\mathrm{SiO}_{4}$ tetrahedron and the network of $\mathrm{SiO}_{4}$ tetrahedra was fixed. The coordination of alkali ions were determined by using MD calculations.

\subsection{Running procedure}

The initial positions of atoms were set up by the obtained model, whose structure was similar to the positions of atoms of $\mathrm{Na}_{2} \mathrm{O} \cdot 2 \mathrm{SiO}_{2}$ glass $^{1,5)}$. The parent cell and cell lengths were shown in Fig. 3. The parent cell consisted of 18 atoms. They were 10 oxygen ions, 4 silicon atoms and 4 potassium ions. These 18 atoms were enclosed in a tetragonal cell. The Si-O bonding length was determined from the first peak of the observed RDF. The increment of time of MD calculations was determined in order to obtain the reasonable configuration of the glass without divergence at the calculation time. By a small stepwise increment of time, $\Delta T=3.3 \times 10^{-15} \mathrm{~s}$, a possible configuration of alkali ions was calculated. In the calculations of this work, a temperature was lowered by reducing the kinetic energy of the atoms in each step and finally to the room temperature, $293 \mathrm{~K}$. After the movement of ions in ions in one step

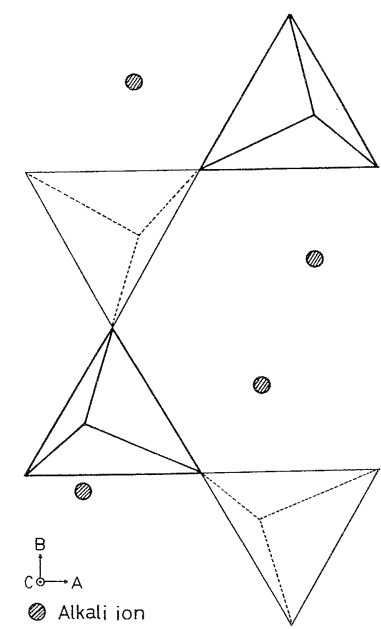

BASIC CELL

Fig. 3. The parent cell of $\mathrm{K}_{2} \mathrm{O} \cdot 2 \mathrm{SiO}_{2}$ glass for molecular dynamics calculations.

The cell lengths were $A=5.24 \AA, B=9.02 \AA, C=$ $6.13 \AA$.

became small enough to be neglected, the calculation was stopped. The calculated RDFs were obtained from the final configuration by using pair function method ${ }^{7)}$. These calculations were carried out on Hitachi M-280 H or Fujitsu M-180 AD.

\section{Analysis and determination of the structures of the disilicate glasses}

\subsection{General aspects of analysis}

Our purpose is to determine the average structure of glasses in the range of $0-5 \AA$. Due to the hygroscopy of potassium disilicate glass, the corresponding crystal structures had not been reported. So we referred other structure of the disilicate glass. $\mathrm{Na}_{2} \mathrm{O} \cdot 2 \mathrm{SiO}_{2}$ crystal has a layer structure and it is natural to assume that a disilicate glass has generally a layer structure too. It has already been clarified that the structure of sodium disilicate glass can be described in terms of sheet structure ${ }^{1,5)}$. In construction of the structural model of the network of potassium disilicate glass, the structure of sodium disilicate glass was referred. In a unit structure, there were four $\mathrm{SiO}_{4}$ tetrahedra and four alkali ions, which formed a part of a six-membered ring. This unit was repeated until the model was large enough to cover the range of 5-10 $\AA$ being calculated. The calculated RDF was obtained by summing up a few hundreds of pair functions, whose positions and peak heights correspond to the distances, the 
numbers and kinds of atomic pairs in the model. In the analysis of the structure of $\mathrm{Li}_{2} \mathrm{O} \cdot 2 \mathrm{SiO}_{2}$ glass, more than one hundred calculations were made by changing the parameters of the structural models until the best agreement between the theoretical and observed RDF was obtained. In the final stage of calculation, we introduced the disorder in accordance with the anticipated structural flexibility.

\section{2 Selection and determination of structural parameters}

The deformation parameters of sheet structure, $A, B, C$ and the shift parameters for stacking layers along the axis, $X, Y$ and $Z$ were shown in Fig.4. Alkali ions were surrounded by four non-bridging oxygen atoms belonging to $\mathrm{SiO}_{4}$ tetrahedra in upper and lower layers and two bridging oxygen atoms bind these tetrahedra in a six-membered ring. In this configuration, alkali ions are in coordination with these oxygen atoms at a distace equal to the sum of the ionic radii. After the structural model of the network was determined, the effect of structural parameters on the calculated RDFs were investigated and the model that showed the best fit with the observed RDF was pursued.

\section{3 Structure of $\mathrm{K}_{2} \mathrm{O} \cdot 2 \mathrm{SiO}_{2}$ glass}

The structural parameters, including shift parameters, were changed so that an alkali ion could be accommodated. The bending angle $A$ in the $z^{-x}$ plane was changed because it had the considerable effects on the state of potassium
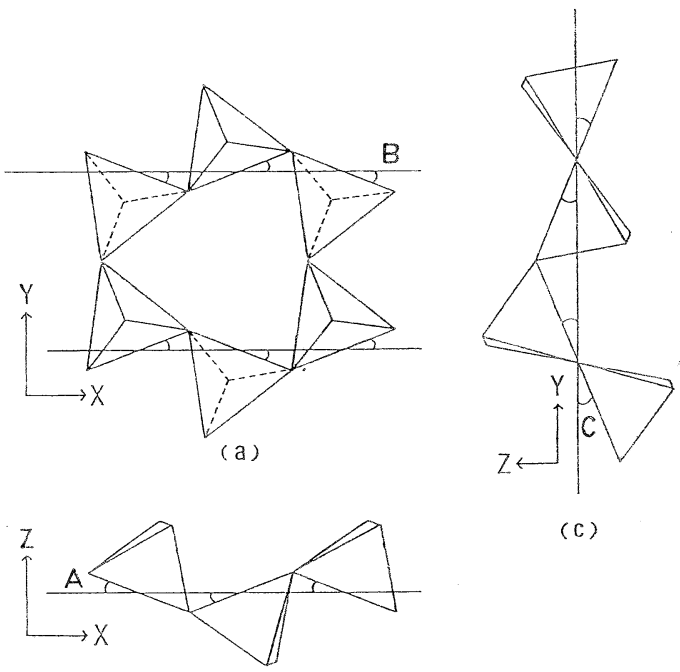

(c)

(b)

Fig. 4. The deformation parameters of the sheet structure. ions. The structures of the networks of alkali silicate glasses with the amount of alkali oxide about $33 \mathrm{~mol} \%$ was mainly determined by the steric factors, i.e. the ionic radii of containing alkali ions. In the study of potassium metasilicate glass ${ }^{8)}$, the bending angles in chains of $\mathrm{SiO}_{4}$ tetrahedra are near to 8 degrees and in the case of sodium disilicate the bending angle $A$ is about 20 degrees. The layer structure in disilicate can be regarded as being formed of metasilicate chains, which were found in the metasilicate glass structures. The arrangement of alkali ions in a sixmembered ring may also be similar to that in metasilicate chains. To take into consideration of these similarity, we determined that the bending angle $A$ was in the range from ca. 8 to 20 degrees. RDFs were calculated for the models constructured with these deformation parameters. The bending angle $B$ in the $x-y$ plane in the range of 0 to 12 degrees has very little effects on the calculated RDF, which was different from the case of $A$. In comparison with the observed RDF and the calculated RDF, it was formed that the bending parameters, $A=12, B=0, C=10$ degrees gave the best agreement. The determined parameters and $\mathrm{Si}-\mathrm{O}$ bonding angles were shown in Table 1. The maximum bonding angles of oxygen atoms in silicate crystals are close to 160 degrees and in silicate glasses a bonding angle close to this value is also more likely than 180 degrees. So a value of $A$ near to 12 degrees may be appropriate. The calculated RDF and observed RDF were shown in Fig. 5.

The good agreement with the observed RDF and the calculated RDF was obtained. After determining the network for the structure model,

Table 1. The results of parameters of $\mathrm{K}_{2} \mathrm{O} \cdot 2 \mathrm{SiO}_{2}$ glass.

\begin{tabular}{|c|c|c|c|}
\hline \multirow[t]{3}{*}{ Bending angle } & $A=12$ & in $z-x$ & plane \\
\hline & $B=\emptyset$ & in $x-y$ & plane \\
\hline & $C=1 \emptyset$ & in $y-z$ & plane \\
\hline \multicolumn{4}{|c|}{ The shift along the axis } \\
\hline & in & stacking & layer \\
\hline & $x$-axis & $1 . \emptyset \mathrm{A}$ & \\
\hline & $y$-axis & $0.3 \mathrm{~A}$ & \\
\hline
\end{tabular}

Si-O-Si bonding angles 


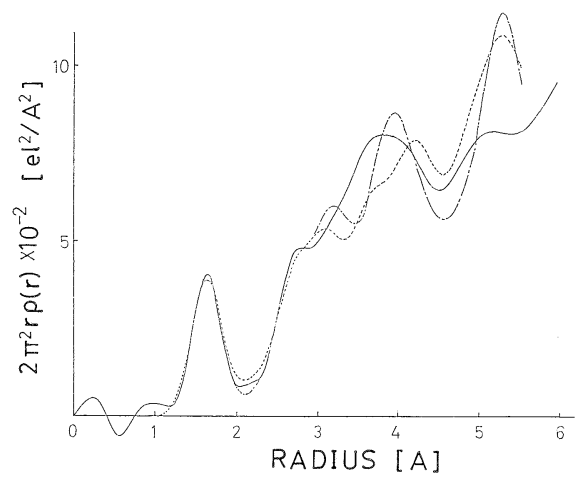

Fig. 5. Calculated and observed radial distribution functions (RDF) of $\mathrm{K}_{2} \mathrm{O} \cdot 2 \mathrm{SiO}_{2}$ glass.

Calculated RDFs were before and after MD calculations.

....... : RDF before MD calculation,

-- : RDF after MD calculation,

: Observed RDF

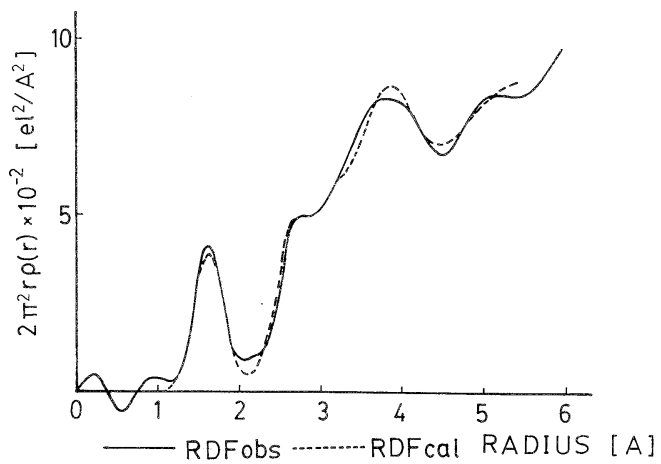

Fig.6. Calculated radial distribution function (RDF) of $\mathrm{K}_{2} \mathrm{O} \cdot 2 \mathrm{SiO}_{2}$ glass after MD calculation.

The disorder was introduced in accordance with the structural flexibility.

Table 2. The change of interatomic distances of $\mathrm{K}_{2} \mathrm{O} \cdot 2 \mathrm{SiO}_{2}$ glass

The interatomic distance of $\mathrm{K}-\mathrm{K}$ was shortened after MD calculation.

\begin{tabular}{lcc}
$\begin{array}{c}\text { Kind } \\
\text { of } \\
\text { atoms }\end{array}$ & Interatomic distance (A) \\
\hline before MD calc. & after MD calc. \\
\hline K-K & 2.538 & 2.619 \\
K-Si & 2.207 & 2.503 \\
& 3.001 & 3.939
\end{tabular}

the MD calculation were carried out. The RDF curves before and after MD calculation were shown in Fig. 6, and the change of interatomic distances were shown in Table 2.

Disorder was introduced as the increase of the peak width similar to the case observed in sodium disilicate glass ${ }^{5}$, which also consisted of layer structure. In this procedure, the peak widths of the interlayer atomic pairs were spread more than those of the intralayer pairs.

\section{4 Structure of $\mathrm{Li}_{2} \mathrm{O} \cdot 2 \mathrm{SiO}_{2}$}

In the analysis of the structure of lithium disilicate glass, MD calculations were not carried out, and the structure was determined only by using the pair function method. The structural parameters were changed so that four lithium ions could be accommodated in a unit cell. At first, the bending angle $A$ in the $z^{-x}$ plane was changed, and the bending angle in chains of $\mathrm{SiO}_{4}$ tetrahedra was near to 0 to 8 degrees in the case of lithium metasilicate glass ${ }^{9}$. In this work, the bending angle $A$ was in the range from ca. 8 to 20. The six-membered ring had a closer structure in comparison with potassium disilicate glass, because the radius of lithium ion is smaller than that of potassium ion. It was formed that the bending parameters, $A=13, B$

Table 3. The results of parameters of $\mathrm{Li}_{2} \mathrm{O} \cdot 2 \mathrm{SiO}_{2}$ glass.

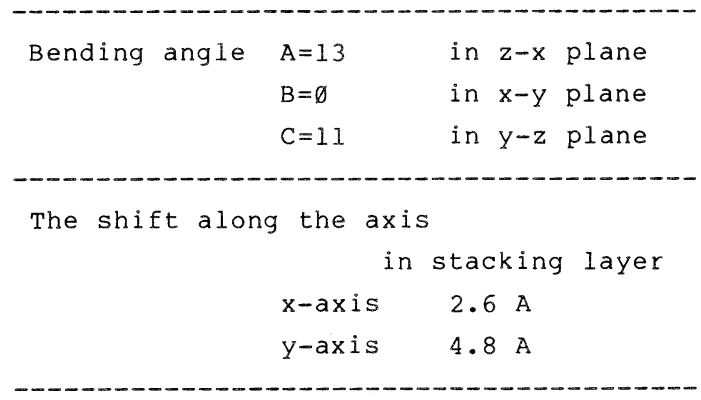

Si-O-Si bonding angles

154 degrees, 167 degrees

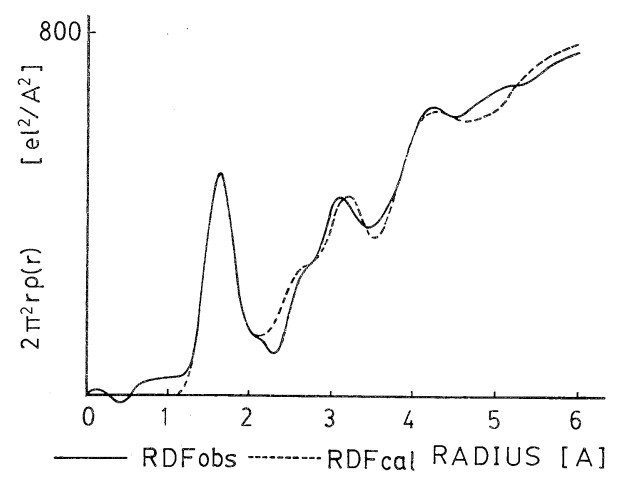

Fig. 7. Calculated radial distribution function (RDF) of the best model and observed RDF of $\mathrm{Li}_{2} \mathrm{O} \cdot 2 \mathrm{SiO}_{2}$ glass. 
$=0, C=11$ degrees gave the best agreement. The determined parameters and Si-O bonding angles were shown in Table 3. Because the bending angle $B$ in $x-y$ plane has very little effects on the calculated RDF, the effect of the bending angle $B$ was not taken into considerations in the structural analysis of $\mathrm{Li}_{2} \mathrm{O} \cdot 2 \mathrm{SiO}_{2}$ glass.

The calculated RDF which showed the best agreement with the observed RDF was shown in Fig. 7. Disorder was introduced as the increase of the peak width similar to that observed in potassium or sodium disilicate glass.

\section{Summary and conclusion}

The most probable structures of $\mathrm{K}_{2} \mathrm{O} \cdot 2 \mathrm{SiO}_{2}$ and $\mathrm{Li}_{2} \mathrm{O} \cdot 2 \mathrm{SiO}_{2}$ glasses were determined by the molecular dynamics calculation and the pair function analysis of the RDF. The following conclusions were obtained :

(1) The structures of both alkali disilicate glasses were shown to consist of layers of sixmembered ring of $\mathrm{SiO}_{4}$ tetrahedra with potassium or lithium ions occupying the positions between them. $\mathrm{K}^{+}$ions and $\mathrm{Li}^{+}$ions were accomodated in the space formed by four or two non-bridging oxygen atoms of upper and lower layers.

(2) When the layer structure of disilicate glass was recognized as a composite of chains of their corresponding metasilicate glasses, the parameters for both silicate structures had close similarities. This means the network structure of alkali silicate glasses with the amount of alkali oxide about 33 mol\%, mainly determined by the steric factors, i.e. the ionic radii of containing alkali ions.

( 3 ) In $\mathrm{K}_{2} \mathrm{O} \cdot 2 \mathrm{SiO}_{2}$ glass, the $\mathrm{MD}$ calculation were effective to obtain the most appropriate configuration and to minimize the efforts of constructing many models. Moreover, the molecular dynamics (MD) calculation can increase some physical meanings of the calculated model.

(4) The result of this work agreed with the presious studies on $\mathrm{Na}_{2} \mathrm{O} \cdot 2 \mathrm{SiO}_{2}$ and $\mathrm{Li}_{2} \mathrm{O}$. $\mathrm{K}_{2} \mathrm{O} \cdot 4 \quad \mathrm{SiO}_{2}$ glasses, which have the layer structures.

\section{References}

1) I. Yasui, H. Hasegawa and M. Imaoka, Phys. Chem. Glasses, 24, 75 (1983).

2) L. V. Woodcock and K. Singer, Trans. Faraday Soc., 67, 12 (1971).

3) T. F. Soules, J. Non-Cryst. Solids, 49, 29 (1982).

4) T.F. Soules and R.F. Busboy, J. Phys. Chem., 75, 969 (1981).

5) M. Imaoka, H. Hasegawa and I. Yasui, Phys. Chem. Glasses, 24, 72 (1983).

6) L. Verlet, Phys. Rev., 159, 98 (1967)

7) B.E. Warren, "X-ray Diffraction", Addison-Wesley (1969).

8) I. Yasui, H. Hasegawa and M. Imaoka, Yogyo-KyokaiShi, 87, 242-48 (1979).

9) H. Hasegawa, K. Adachi, I. Yasui and M. Imaoka, Yogyo-Kyokai-Shi, 88, 271-77 (1980). 